\title{
Detection Efficiency Modelling and Joint Activity and Attenuation Reconstruction in non-TOF 3D PET from Multiple-Energy Window Data
}

\author{
Ludovica Brusaferri, Elise C. Emond, Alexandre Bousse, Robert Twyman, Student Member, IEEE, Alexander C. \\ Whitehead, Student Member, IEEE, David Atkinson, Sébastien Ourselin, Brian F. Hutton, Senior Member, IEEE, \\ Simon Arridge and Kris Thielemans, Senior Member, IEEE
}

\begin{abstract}
Emission-based attenuation correction (AC) methods offer the possibility of overcoming quantification errors induced by conventional MR-based approaches in PET/MR imaging. However, the joint problem of determining AC and the activity of interest is strongly ill-posed in non-TOF PET. This can be improved by exploiting the extra information arising from low energy window photons, but the feasibility of this approach has only been studied with relatively simplistic analytic simulations so far. This manuscript aims to address some of the remaining challenges needed to handle realistic measurements; in particular, the detection efficiency ("normalisation") estimation for each energy window is investigated. An energy-dependent detection efficiency model is proposed, accounting for the presence of unscattered events in the lower energy window due to detector scatter. Geometric calibration factors are estimated prior to the reconstruction for both scattered and unscattered events. Different reconstruction methods are also compared. Results show that geometric factors differ markedly between the energy windows and that our analytical model correspond in good approximation to Monte Carlo simulation; the multiple energy window reconstruction appears sensitive to input/model mismatch. Our method applies to Monte Carlo generated data but can be extended to measured data. This study is restricted to single scatter events.
\end{abstract}

Index Terms-Positron Emission Tomography, PET-MR, Attenuation Estimation, Iterative Methods, Monte Carlo, Optimisation, Scatter.

\section{INTRODUCTION}

This work was supported by Siemens Healthineers, the UCL Impact Scheme, NIHR UCLH Biomedical Research Centre, the EPSRC-funded UCL Centre for Doctoral Training in Medical Imaging (EP/L016478/1) and the UCL Institute of Healthcare Engineering. Elise Emond was supported by GlaxoSmithKline (BIDS3000030921). Robert Twyman and Alexander C. Whitehead were supported by GE Healthcare.

Ludovica Brusaferri was with the Institute of Nuclear Medicine, University College London, London NW1 2BU, UK and is now with Athinoula A. Martinos Center for Biomedical Imaging, Harvard Medical School, 02129, Boston, MA, US.

Elise C. Emond, Robert Twyman, Alexander C. Whitehead, Brian Hutton and Kris Thielemans are with the Institute of Nuclear Medicine, University College London, London NW1 2BU, UK.

Alexandre Bousse was with the Institute of Nuclear Medicine, University College London, London NW1 2BU, UK and is now with the Laboratoire de Traitement de l'Information Médicale - LATIM, INSERM, U1101, Université de Bretagne Occidentale, Brest, France.

David Atkinson is with the Centre for Medical Imaging, University College London, London W1W 7TS, UK.

Sébastien Ourselin is with the School of Biomedical Engineering and Imaging Sciences, King's College London, London SE1 7EH, UK.

Simon Arridge is with the Department of Computer Science, University College London, London WC1E 6BT, UK.
$\mathbf{M}$ ULTI-MODALITY scanners have recently made great strides towards improving reliability and accuracy of medical imaging. Hybrid Positron Emission Tomography (PET) and Computed Tomography (CT) (PET/CT) scanners make use of the anatomical information from the CT for the attenuation correction of the PET activity distribution and also for other clinical staging and diagnostic processes [1]. At present, PET/CT is generally the modality of choice in many clinical conditions. Additionally, new multi-modality PET scanners have become available in the last decade, such as PET/MR scanners. Where PET/CT performs sequential PETCT data acquisitions, a key strength of PET and Magnetic Resonance (MR) (PET/MR) is the capability for simultaneous data acquisition of its two modalities. Furthermore, PET/MR combines the unique features of MR (i.e. excellent soft tissue contrast, diffusion-weighted imaging, functional Magnetic Resonance Imaging (fMRI), and other specialised sequences) with the quantitative functional information that is provided by PET [1], [2]. Some specific clinical applications - especially in brain studies - may favour PET/MR over PET/CT; such as patients undergoing repeated imaging in a limited time with the need to keep radiation dose as low as possible.

A well-known challenge of PET/MR is however the difficulty to find an accurate and reliable method to estimate PET attenuation maps without relying on a CT acquisition or without a transmission scan. PET attenuation coefficients depend on the electron density of the tissues, whereas the MR signal is related to both proton density and longitudinal (T1) and transverse (T2 and $\mathrm{T} 2 *$ ) magnetisation relaxation times. At present, there is no straightforward transformation able to map MR intensities into PET attenuation values [3].

Segmentation-based methods are the most frequently used approaches for PET/MR attenuation correction in clinical practice. The MR image is usually segmented into different tissue or attenuation areas (i.e., bone, fat, lung, and air) and population-based attenuation coefficients are assigned to these regions. The main inaccuracies of this approach arise from the inability to account for variations in attenuation coefficients between subjects [2]. For tissues such as the lung, where attenuation coefficient variation is high [4], this can significantly affect PET quantification [5]. Lung PET/MR attenuation correction is still an ongoing field of research.

Several techniques estimate the attenuation image from the PET emission data - known as emission-based methods - 
sometimes incorporating MR images as prior information. These techniques might also be able to overcome variations in lung density due to respiration [5], [6]. A common emissionbased method to obtain the attenuation map from PET data is Maximum Likelihood reconstruction of Activity and Attenuation (MLAA) [7]. Unfortunately, a stable solution for this approach only exists when Time-Of-Flight (TOF) information is available, in which case the activity can then be determined up to an unknown scaling factor [8]. This global constant can be found by imposing known attenuation values in certain regions, such as soft tissue [9].

In non-TOF MLAA instead, the estimated activity and attenuation maps still suffer from cross-talk artefacts [10], where features of the activity map propagate into the attenuation map and vice versa. Therefore, further solutions need to be found for reconstructing accurate PET images from non-TOF data.

Several authors have attempted to overcome cross-talk in non-TOF PET by exploiting the energy information contained in multi-energy window acquired data. This was first investigated in Single Photon Emission Computed Tomography (SPECT) [11], [12] and then extended to the case of PET [13][15]. However, these studies were conducted based on detection efficiency models ignoring detector scatter and neglecting the presence of true events in the low-energy windows. As a consequence, the aforementioned PET approaches were only tested on relatively simplistic analytical simulations and did not investigate the effects of mismatch between the acquisition model and the actual measurement process. Indeed, the performance of an iterative reconstruction method can strongly depend on the accuracy and reliability of the forward model of the data.

For energy-based PET image reconstruction methods, an important factor is played by the dependency of the detector response on the energy of the incoming gamma photons. A common method to model the energy response of PET detectors in the photopeak window is to use an energydependent Gaussian broadening [16], [17]. However, for either wider energy windows or in the case of energy windows placed below the photopeak, this approximation is inaccurate. In practice, scatter of the gamma photons within the scintillation crystal will result in partial energy deposition. Consequently, the presence of unscattered events in the low energy windows can occur [18].

The problem of taking these differences in detection efficiency into account for a photopeak energy window was first investigated in [18] where different normalisation approaches were proposed. This study showed that scatter estimation and detector-efficiency normalisation cannot be treated as independent issues. It also opens several questions on the importance of normalisation factors for multiple energy windows.

The current work aims to address some of the challenges encountered in multiple energy windows PET reconstruction. Overall, the following improvements on previous research are provided: (i) incorporation of detector scatter in the energydependent detection efficiency model, (ii) accounting for the presence of unscattered events in the low energy windows, (iii) estimation of normalisation sinograms for each energy window prior to the reconstruction, (iv) realistic input/output for the reconstruction algorithm, (v) investigation of the effects of inaccuracies in the detection efficiency model.

This manuscript is organised as follows. We first cover the mathematical theory relevant to the framework, then give an overview of the proposed methodology (with some details in the appendices). We finally present results from GATE Monte Carlo simulated data and provide a comparison of the proposed method against MLAA from a single energy window acquisition. Similar to previous published work [15], [19], the current study is restricted to single scatter only.

\section{THEORY}

\section{A. Statistical model of the measured data}

The primary assumption of this work is that the observed counts $\boldsymbol{g}_{v w}$ in each energy window pair $(v, w) \in\left\{1, \ldots, n_{\mathrm{E}}\right\}^{2}$ can be described as a sum of independent Poisson processes with expected value $\overline{\boldsymbol{g}}_{v w}$ :

$$
\overline{\boldsymbol{g}}_{v w}(\boldsymbol{\lambda}, \boldsymbol{\mu})=\overline{\boldsymbol{g}}_{v w}^{\mathrm{unsc}}(\boldsymbol{\lambda}, \boldsymbol{\mu})+\overline{\boldsymbol{g}}_{v w}^{\mathrm{sc}}(\boldsymbol{\lambda}, \boldsymbol{\mu})+\overline{\boldsymbol{g}}_{v w}^{\mathrm{r}}
$$

where $\boldsymbol{\lambda} \in \mathbb{R}^{\mathrm{n}_{\mathrm{v}}}$ and $\boldsymbol{\mu} \in \mathbb{R}^{\mathrm{n}_{\mathrm{v}}}$ are vectors that represent the activity and attenuation distributions of the object, respectively, $\overline{\boldsymbol{g}}_{v w}^{\mathrm{unsc}}$ denotes the expected unscattered events, $\overline{\boldsymbol{g}}_{v w}^{\mathrm{sc}}$ indicates the expected single scatter events and $\overline{\boldsymbol{g}}_{v w}^{r}$ is a background term incorporating randoms. Both $\overline{\boldsymbol{g}}_{v w}^{\mathrm{unsc}}$ and $\overline{\boldsymbol{g}}_{v w}^{\mathrm{sc}}$ are formulated below.

1) Unscattered Events: The expected unscattered events $\overline{\boldsymbol{g}}_{v w}^{\mathrm{unsc}}(\boldsymbol{\lambda}, \boldsymbol{\mu})$ are modelled as follows:

$$
\overline{\boldsymbol{g}}_{v w}^{\mathrm{unsc}}(\boldsymbol{\lambda}, \boldsymbol{\mu})=\boldsymbol{A}_{v w}(\boldsymbol{\mu}) \boldsymbol{\lambda}
$$

where $\boldsymbol{A}_{v w}(\boldsymbol{\mu}) \in \mathbb{R}_{+}^{n_{\mathrm{D}} \times n_{\mathrm{V}}}$ is a linear transformation mapping from image space to data space, describing the probability that an emission occurring in voxel $u \in\left\{1, \ldots, n_{\mathrm{V}}\right\}$ is detected by a pair of detectors $(h, k) \in\left\{1, \ldots, n_{\mathrm{D}}\right\}^{2}$ for a given energy window pair $(v, w)$ :

$$
\boldsymbol{A}_{v w}(\boldsymbol{\mu})=\boldsymbol{\Psi}_{v w}(511,511) \boldsymbol{D}[\exp (-\boldsymbol{L} \boldsymbol{\mu})] \boldsymbol{L}
$$

with $\boldsymbol{D}[\cdot]$ indicating a diagonal matrix, $\boldsymbol{\Psi}_{v w}(511,511)$ being the diagonal matrix of efficiency factors for $511 \mathrm{keV}$ incoming photons and $\boldsymbol{L}$ computing the line integral operation. In this work, dead-time effects are not included in the model.

2) Scattered Events: A detailed formulation for the expected single scatter events $\overline{\boldsymbol{g}}_{v w}^{\text {sc }}(\boldsymbol{\theta})$ now follows. See [15] for more information. For computational efficiency, we will model the scatter in low resolution [16], [20], [21].

We introduce here the variable $\boldsymbol{\theta}=(\boldsymbol{\lambda}, \boldsymbol{\mu})$. Let $\boldsymbol{P} \in$ $\mathbb{R}_{+}^{n_{\mathrm{D}} \times n_{\mathrm{D}}{ }^{\prime}}$ and $\boldsymbol{R} \in \mathbb{R}_{+}^{n_{\mathrm{V}}{ }^{\prime} \times n_{\mathrm{V}}}$ be prolongation and restriction operators, respectively. Both operators are linear. The former maps a sinogram from low resolution ( $n_{\mathrm{D}}{ }^{\prime}$ total detector pairs) to high resolution ( $n_{\mathrm{D}}$ total detector pairs). The latter maps an image from high resolution ( $n_{\mathrm{V}}$ voxels) to low resolution $\left(n_{\mathrm{V}}{ }^{\prime}\right.$ voxels). For simplicity, we will write $\boldsymbol{R} \boldsymbol{\theta}$ for $(\boldsymbol{R} \boldsymbol{\lambda}, \boldsymbol{R} \boldsymbol{\mu})$. Also let $n_{\mathrm{D}}{ }^{\prime}=\gamma n_{\mathrm{D}}$ and $n_{\mathrm{V}}=\eta n_{\mathrm{V}}{ }^{\prime}$, with $\gamma$ and $\eta$ defining the downsampling and upsampling factors in sinogram and image space, respectively. Then, the scatter component $\overline{\boldsymbol{g}}_{v w}^{\mathrm{sc}}(\boldsymbol{\theta})$ is given by the following relationship: 


$$
\overline{\boldsymbol{g}}_{v w}^{\mathrm{sc}}(\boldsymbol{\theta})=\boldsymbol{P} \boldsymbol{S}_{v w}(\tilde{\boldsymbol{\theta}})
$$

where:

$$
\tilde{\boldsymbol{\theta}}=\boldsymbol{R} \boldsymbol{\theta}
$$

and $\boldsymbol{S}_{v w}(\tilde{\boldsymbol{\theta}}) \in \mathbb{R}_{+}^{n_{\mathrm{D}}{ }^{\prime} \times n_{\mathrm{V}}{ }^{\prime}}$ is the forward operator computing the expected (single) scatter at an energy window pair $(v, w)$ for every pair of (down-sampled) detectors $(i, j) \in$ $\left\{1, \ldots, n_{\mathrm{D}}\right\}^{2}$. An element $(i, j)$ is such that:

$$
\left[\boldsymbol{S}_{v w}(\tilde{\boldsymbol{\theta}})\right]_{i j}=\sum_{s=1}^{n_{\mathrm{S}}} \tilde{g}_{v w i j s}^{\mathrm{sc}}(\tilde{\boldsymbol{\theta}})
$$

where $s$ is an index over scatter points and $n_{\mathrm{S}}$ is the total number of scatter points. Details on the scatter model, including the proposed energy-dependent detection efficiency (now accounting for detector scatter) can be found in appendix A and B.

\section{B. Objective Function for joint activity and attenuation esti- mation}

The log-likelihood of the measured data $\boldsymbol{g}_{v w}$ at a certain energy window pair $(v, w)$ is given by:

$$
\begin{aligned}
& \mathcal{L}\left(\boldsymbol{g}_{v w} \mid \overline{\boldsymbol{g}}_{v w}(\boldsymbol{\lambda}, \boldsymbol{\mu})\right)= \\
& \sum_{h=1}^{n_{\mathrm{D}}} \sum_{k=1}^{n_{\mathrm{D}}}\left(g_{v w h k} \log \bar{g}_{v w h k}(\boldsymbol{\lambda}, \boldsymbol{\mu})-\bar{g}_{v w h k}(\boldsymbol{\lambda}, \boldsymbol{\mu})\right)
\end{aligned}
$$

The joint maximum-likelihood reconstruction of $\boldsymbol{\lambda}$ and $\boldsymbol{\mu}$ is achieved by solving:

$$
(\hat{\boldsymbol{\lambda}}, \hat{\boldsymbol{\mu}})=\underset{\boldsymbol{\lambda} \geq \mathbf{0}, \boldsymbol{\mu} \geq \mathbf{0}}{\arg \max } \mathcal{L}^{\mathrm{tot}}(\boldsymbol{g} \mid \overline{\boldsymbol{g}}(\boldsymbol{\lambda}, \boldsymbol{\mu}))
$$

with:

$$
\mathcal{L}^{\text {tot }}=\sum_{v=1}^{n_{\mathrm{E}}} \sum_{w=1}^{n_{\mathrm{E}}} \mathcal{L}_{v w}
$$

The gradient of the objective function $\mathcal{L}^{\text {tot }}$ is given in Appendix C.

\section{MethodS}

\section{A. Maximum Likelihood Estimation of the Detection Efficiency Factors}

Here we propose a method to estimate detection efficiency correction factors for the normalisation of PET emission data from multiple-energy windows, applicable to Monte Carlo data. We extended [22] by accounting for the variation in detection efficiency for each energy window $v w$ of both scattered and unscattered events:

$$
\overline{\boldsymbol{g}}_{v w}=\overline{\boldsymbol{g}}_{v w}^{\mathrm{unsc}}\left(\boldsymbol{\Psi}_{v w}^{\mathrm{unsc}}\right)+\overline{\boldsymbol{g}}_{v w}^{\mathrm{sc}}\left(\boldsymbol{\Psi}_{v w}^{\mathrm{sc}}\right)
$$

where:

$$
\begin{aligned}
\overline{\boldsymbol{g}}_{v w}^{\mathrm{unsc}}\left(\boldsymbol{\Psi}_{v w}^{\mathrm{unsc}}\right) & =\boldsymbol{\Psi}_{v w}^{\mathrm{unsc}} \odot \overline{\boldsymbol{f}}_{v w}^{\mathrm{unsc}}, \\
\overline{\boldsymbol{g}}_{v w}^{\mathrm{sc}}\left(\boldsymbol{\Psi}_{v w}^{\mathrm{sc}}\right) & =\boldsymbol{\Psi}_{v w}^{\mathrm{sc}} \odot \overline{\boldsymbol{f}}_{v w}^{\mathrm{sc}},
\end{aligned}
$$

$\overline{\boldsymbol{f}}_{v w}$ is the forward projection that includes an energydependent detection efficiency model (Appendix A) and $\odot$ denotes element-wise multiplication. The efficiency factors $\boldsymbol{\Psi}_{v w}^{\mathrm{unsc}}$ and $\boldsymbol{\Psi}_{v w}^{\mathrm{sc}}$ are defined as:

$$
\begin{aligned}
\boldsymbol{\Psi}_{i j}^{\text {unsc }} & =\epsilon_{i} \epsilon_{j} \tau_{i j}^{\text {unsc }} \\
\Psi_{i j}^{\text {sc }} & =\epsilon_{i} \epsilon_{j} \tau_{i j}^{\text {sc }}
\end{aligned}
$$

where $(i, j)$ is a detector pair in the scanner, $\epsilon_{i} \epsilon_{j}$ indicates crystal efficiency factors (over-and-above the energy-dependent model) and $\tau_{i j}$ are geometric factors accounting for the change in efficiency due to the radial position of the detector pair and relative position of each detector pair within a block; it also depends on the angle of incidence of the incoming photon, which is the reason why we differentiate between $g_{i j}^{\text {unsc }}$ and $g_{i j}^{\mathrm{sc}}$.

In the following, we will refer to $\frac{1}{\Psi}$ as normalisation factors and $\Psi$ as efficiency factors.

Now let us assume in this section that we can identify if an event has been scattered or not, such as feasible in Monte Carlo data. Then, $\boldsymbol{g}_{v w}^{\mathrm{unsc}}$ and $\boldsymbol{g}_{v w}^{\mathrm{sc}}$ denote respectively the measured unscattered and scatter events from a Monte Carlo simulation. A $\log$-likelihood function $\mathcal{L}$ can be then defined, denoting the probability that measured data $\boldsymbol{g}$ are observed, given a known object and the (unknown) efficiency factors $\boldsymbol{\Psi}$. By extending [22], the efficiency factors can be estimated for each energy window pair $(v, w)$ with a ML estimation:

$$
\begin{gathered}
\hat{\boldsymbol{\Psi}}_{v w}^{\mathrm{unsc}}=\underset{\mathbf{\Psi}_{v w}^{\mathrm{unsc}}}{\arg \max }\left[\mathcal{L}_{v w}\left(\boldsymbol{g}_{v w}^{\mathrm{unsc}} \mid \overline{\boldsymbol{g}}_{v w}^{\mathrm{unsc}}\left(\boldsymbol{\Psi}_{v w}^{\mathrm{unsc}}\right)\right)\right] \\
\hat{\boldsymbol{\Psi}}_{v w}^{\mathrm{sc}}=\underset{\mathbf{\Psi}_{v w}^{\mathrm{sc}}}{\arg \max }\left[\mathcal{L}_{v w}\left(\boldsymbol{g}_{v w}^{\mathrm{sc}} \mid \overline{\boldsymbol{g}}_{v w}^{\mathrm{sc}}\left(\boldsymbol{\Psi}_{v w}^{\mathrm{sc}}\right)\right)\right]
\end{gathered}
$$

Please note that in the GATE simulations used in this manuscript, the efficiencies $\epsilon_{i} \epsilon_{j}$ of all the crystals were identical. Therefore, the optimisation problem reduces to the estimation of the geometric factors. Furthermore, in agreement with [22] we imposed symmetry between blocks, allowing us to reduce noise in the estimated normalisation factors.

\section{B. Image Reconstruction Algorithms}

The following section covers the joint reconstruction algorithms used in this manuscript, giving an overview of both multiple and single energy window approaches.

1) Multiple Energy Window Approach:

a) aMLAA-EB-S: The cost function is obtained by summing the log-likelihoods of each energy window pair (9). The activity and the attenuation estimates are updated using an alternating scheme using Bounded Limited-memory Broyden-Fletcher-Goldfarb-Shanno (LBFGSB) optimisation [23] in each step:

$$
\begin{gathered}
\boldsymbol{\mu}^{k+1}=\boldsymbol{\mu}^{k}-\xi_{\boldsymbol{\mu}} \boldsymbol{B}_{\boldsymbol{\mu}} \nabla_{\boldsymbol{\mu}}\left[\mathcal{L}^{\mathrm{tot}}\left(\boldsymbol{\lambda}^{k}, \boldsymbol{\mu}^{k}\right)-\beta_{\boldsymbol{\mu}} \boldsymbol{U}_{\boldsymbol{\mu}}\left(\boldsymbol{\mu}^{k}\right)\right] \\
\boldsymbol{\lambda}^{k+1}=\boldsymbol{\lambda}^{k}-\xi_{\boldsymbol{\lambda}} \boldsymbol{B}_{\boldsymbol{\lambda}} \nabla_{\boldsymbol{\lambda}}\left[\mathcal{L}^{\mathrm{tot}}\left(\boldsymbol{\lambda}^{k}, \boldsymbol{\mu}^{k+1}\right)-\beta_{\boldsymbol{\lambda}} \boldsymbol{U}_{\boldsymbol{\lambda}}\left(\boldsymbol{\lambda}^{k}\right)\right]
\end{gathered}
$$

where $\nabla_{\lambda} \mathcal{L}^{\text {tot }}$ and $\nabla_{\mu} \mathcal{L}^{\text {tot }}$ are the gradients of the objective function with respect to the current estimate of the activity and 
attenuation image, respectively, $\boldsymbol{B}_{\boldsymbol{\lambda}}$ and $\boldsymbol{B}_{\boldsymbol{\mu}}$ approximate the inverse Hessian matrix of $\mathcal{L}$, in $\boldsymbol{\lambda}$ and $\boldsymbol{\mu}$ respectively, $\xi_{\boldsymbol{\mu}}$ and $\xi_{\boldsymbol{\lambda}}$ are the step-size, and $\boldsymbol{U}(\boldsymbol{\mu})$ are penalty functions weighted by global strength parameters $\beta_{\boldsymbol{\lambda}}$ and $\beta_{\boldsymbol{\mu}}$, respectively. For the results presented in this paper, the Parallel Level Sets (PLS) prior [24] was chosen as penalty term (Sec. IV-F2).

Pseudo code for Energy-Based Alternating Maximum Likelihood reconstruction of Activity and Attenuation with photopeak Scatter re-estimation (aMLAA-EB-S) is shown in Algorithm 1.

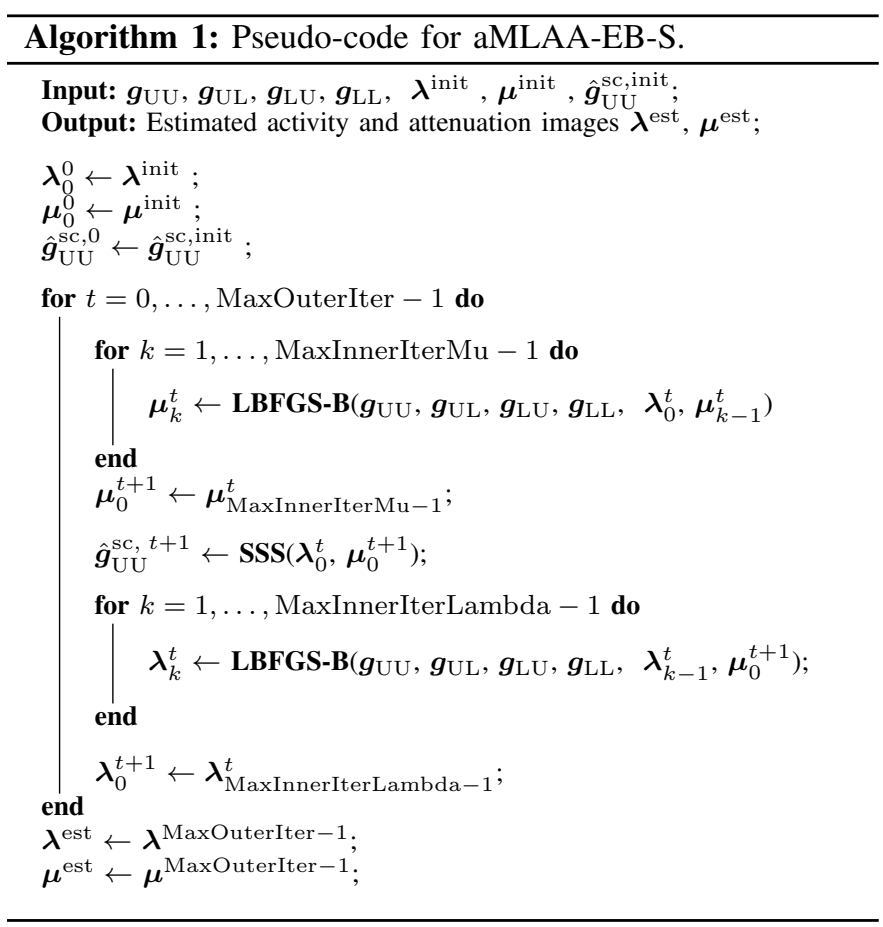

. Please note that $\mathrm{U}$ indicates the upper energy window and $\mathrm{L}$ the lower energy window with $n_{\mathrm{E}}=2$.

2) Single Energy Window Approach:

a) $a M L A A-S$ : The cost function of alternating Maximum Likelihood reconstruction of Activity and Attenuation with photopeak Scatter re-estimation (aMLAA-S) is obtained from (9) with $n_{\mathrm{E}}=1$. The optimisation framework follows the one from aMLAA-EB-S.

b) LBFGS-AC: An LBFGS emission reconstruction using the true attenuation map (LBFGS-AC) was used as goldstandard comparison. The algorithm outputs an estimate of the activity image from the following inputs: (i) ground truth attenuation image $\boldsymbol{\mu}^{\text {true }}$, (ii) photopeak window projection data $\boldsymbol{g}_{\mathrm{UU}}$. The photopeak scatter is re-estimated during iterations.

c) LBFGS-MRAC: An LBFGS emission reconstruction using the MRAC attenuation map (LBFGS-MRAC) was also computed. The algorithm outputs an estimate of the activity image from the following inputs: (i) MRAC attenuation image $\boldsymbol{\mu}^{\text {init }}$, (ii) photopeak window projection data $\boldsymbol{g}_{\mathrm{UU}}$. The photopeak scatter is re-estimated during iterations. The $\boldsymbol{\mu}$-map is not updated over iterations as it would be the case for activity reconstruction only.

\section{EVALUATION}

\section{A. GATE simulation of a Siemens mMR Biograph}

Simulations of a PET scanner similar to the Siemens mMR Biograph [25] were conducted based on a GEANT Application Tomography Emission (GATE) Monte Carlo simulation [26] (GATE 8.0 and Geant4 10.4). The PET system includes 64 rings, with each containing 56 blocks. Each block consists of 1x8x9 Lutetium Oxyorthosilicate (LSO) crystals. Please note that the real mMR uses a $1 \times 8 \times 8$ block; therefore, the gaps between the detector blocks are artificially removed in our simulations. Eight blocks are placed in the axial direction, resulting in a total of 64 rings and 504 detector per ring. The transaxial Field Of View (FOV) is $65.6 \mathrm{~cm}$. The energy resolution is set to $14.5 \%$, according to the mMR characteristics.

Recent PET/CT scanners - such as the Siemens Biograph Vision and the GE Discovery MI - have the capability of a multiple energy acquisition in experimental mode. In the following, we have simulated the possibility of measuring two energy windows. Specifically, $\mathrm{U}=460-570 \mathrm{keV}$, $\mathrm{L}=350-460 \mathrm{keV}$. Therefore, the list-mode GATE files were unlisted into four sinograms $\left(\boldsymbol{g}_{\mathrm{UU}}, \boldsymbol{g}_{\mathrm{UL}}, \boldsymbol{g}_{\mathrm{LU}}, \boldsymbol{g}_{\mathrm{LL}}\right)$, as would be the case for a two-energy window acquisition. For this study, $\boldsymbol{g}_{\mathrm{LL}}=0$. The unlisting procedure was implemented on a private fork of STIR [27]. Please note that only direct sinograms are used in the results of this manuscript.

\section{B. STIR acquisition modelling}

We used a ray-tracing model with 344 tangential positions, 252 views, 64 rings and 504 detectors per ring.

The scatter component is computed at low resolution with 21 views, 31 tangential positions and 8 rings and then upsampled with a linear interpolation to full resolution via the operator $\boldsymbol{P}$ (Sec. II-A). Simulations use in-plane detector pairs only but out-of-plane scatter points were included. The energy resolution was set to $14 \%$ for $511 \mathrm{keV}$ photons.

\section{Energy-Dependent Detection Efficiency Fitting}

A point source of $511 \mathrm{keV}$ located at the centre of the scanner was used to generate the training set data.

The Python SciPy [28] library was used to fit the proposed detection efficiency model (Appendix A) to the GATE simulation. Once the parameters of the model were fit, the model was tested with a GATE data-set from a point source emitting $370 \mathrm{keV}$ photons.

\section{Estimation of Efficiency Factors}

A 3D volume of a cylindrical phantom with elliptical section (covering the region occupied by the XCAT 3D volume) was generated. Image dimension and voxel size were respectively $120 \times 120 \times 64$ and $0.4 \times 0.4 \times 0.40625 \mathrm{~cm}^{3}$. The attenuation material was set to lung tissue defined in the default GATE database $\left(\boldsymbol{\mu}_{511}=0.0247 \mathrm{~cm}^{-1}\right)$.

A maximum-likelihood estimation of efficiency factors for PET detectors was conducted for both scattered and unscattered events: $\boldsymbol{\Psi}_{v w}^{\mathrm{unsc}}$ and $\boldsymbol{\Psi}_{v w}^{\mathrm{sc}}$. In particular, they were obtained by comparing GATE simulated data (Sec. IV-A) to an estimate 


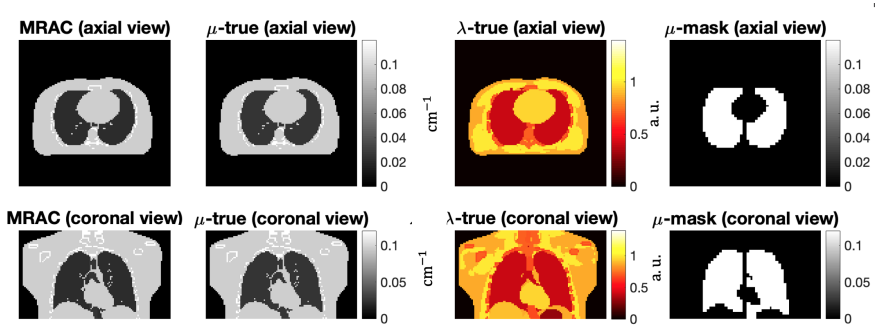

Fig. 1. 3D XCAT Thorso Phantom. From left to right: MRAC with $15 \%$ decreasing attenuation value (See Sec. IV-F3), true attenuation image, true activity image, attenuation mask. First row: axial view. Second row: coronal view.

of the projection data using the forward model in Sec. II-A, obtained with STIR (Sec. IV-B).

The efficiency factors of the true counts in the region outside the support of the activity image are undefined and were therefore set to 0 .

\section{E. XCAT Simulation - Comparison in Sinogram Space}

A 3D volume from the XCAT torso phantom [29] was generated, cropped to a 120x120x64 matrix with voxel size of $0.4 \times 0.4 \times 0.40625 \mathrm{~cm}^{3}$. Both axial and coronal views of the phantom are shown in Fig. 1. The distribution represents the normalised radioactivity distribution corresponding to a static FDG acquisition.

GATE simulations from the input XCAT voxelised image were performed as in Sec. IV-A. Sinograms for UU, UL and LU from GATE simulations were obtained via unlisting the GATE root file into multiple energy window sinograms. STIR sinograms were obtained as explained in Sec. IV-B from the forward model in Sec. II-A and the detection efficiency model in Appendix A.

\section{F. Reconstruction parameters}

The maximum number of inner iterations for the attenuation update (MaxInnerIterMu) was set to 5, whilst the one for the activity (MaxInnerIterLambda) was set to 12 . The maximum number of outer iterations was set to 30. Limited-memory Broyden-Fletcher-Goldfarb-Shanno (LBFGS) stopping criteria were set to the default values for the implementation from [30]. In the current results, lung segmentation was incorporated in the algorithm by only updating the attenuation values within the inner cylinder/lung mask during iterations. The mask extends beyond the exact lung edges (Fig. 1). This constraint is not used for the emission update, for which we only assumed to know the support of the activity image as in [15].

1) Heuristic scaling factor: A heuristic global scaling factor $\zeta=0.93$ was incorporated in the low-energy window normalisation so that the ratio between the expected and measured counts was equal to 1 . The reconstruction was performed both with and without scaling factor to assess the effect of model mismatch.

2) Regularisation parameters: The MRAC (Fig. 1) image was used as anatomical image for the PLS prior [24].

For aMLAA-EB-S reconstructions, the PLS prior was used on both activity and attenuation image estimates. The penalty strength $\beta_{\boldsymbol{\mu}}$ of the PLS prior on the attenuation image was set to 80 ; the penalty strength $\beta_{\boldsymbol{\lambda}}$ of the PLS prior on the activity image was assigned to the value or 5. Smoothing parameters in [24] were both set to 0.03 .

For reconstructions from UU windows only (aMLAA-S, LBFGS-AC and LBFGS-MRAC), the penalty strength was reduced by a factor $f=\frac{\mathcal{L}_{\mathrm{UU}}}{\mathcal{L}_{\mathrm{UU}}+\mathcal{L}_{\mathrm{UL}}+\mathcal{L}_{\mathrm{LU}}}$. The log-likelihood values were computed at the ground truth images.

3) Initial conditions: The initial attenuation image ("MRAC") was generated by decreasing the lung attenuation values by $15 \%$ with respect to the ground truth. The attenuation map was perfectly aligned with the activity image.

The reconstruction was initialised with an activity estimate obtained by iterating between OSEM ( 3 subsets 100 subiterations) and SSS [20] for one outer iteration. The activity image thus obtained was postfiltered with a Gaussian filter with a FWHM of $5 \mathrm{~mm}$.

4) Analysis: The performance evaluation of the reconstruction methods was conducted in terms of relative percentage error with respect to the ground truth images. A visualisation of the bias in the reconstructed image was used as initial assessment. Further investigations were conducted in terms of Mean Percentage Error (MPE) of the estimated images over iterations in different ROIs: (i) 10x10x5 ROI placed within the right lung not overlapping lung structures, (ii) the whole image.

\section{REsults}

\section{A. Energy-Dependent Detection Efficiency Fitting}

Table I reports the values of the parameters obtained by fitting the point source data $(511 \mathrm{keV})$ to the model in Appendix A. In particular, the model is given by the sum of four contributions, respectively (i) Photoelectric Effect, (ii) Compton Scattering, (iii) Flat Continuum, (iv) Exponential Tail. $Z_{\text {eff }}$ indicates the atomic number of the single crystal element and the remaining parameters scale the amplitude and the centre of each of the aforementioned components. See Appendix A for further details.

\begin{tabular}{|cc|}
\hline \multicolumn{2}{|c|}{ Fitted Parameters } \\
\hline $\mathrm{Z}_{\text {eff }}$ & 66 \\
\hline FWHM $(511 \mathrm{keV})$ & 0.14 \\
\hline$H_{1}$ & $0.94010^{26}$ \\
\hline$H_{2}$ & $0.710^{1}$ \\
\hline$H_{3}$ & $0.26010^{2}$ \\
\hline$p_{1}$ & $0.59810^{-2}$ \\
\hline$p_{2}$ & $0.29610^{2}$ \\
\hline$p_{3}$ & $-0.81710^{-2}$ \\
\hline \multicolumn{2}{|c}{ TABLE I } \\
\hline
\end{tabular}

FITTED PARAMETERS FOR THE PROPOSED DETECTION EFFICIENCY MODEL, OBTAINED FROM A MONTE CARLO SIMULATION OF A POINT SOURCE EMITTER.

Fig. 2 shows the model versus the training and test data sets, respectively. The Normalized Root-Mean-Square Deviation (NRMSD) was found to be equal to 0.015 for the training set and equal to 0.03 for the testing set. 


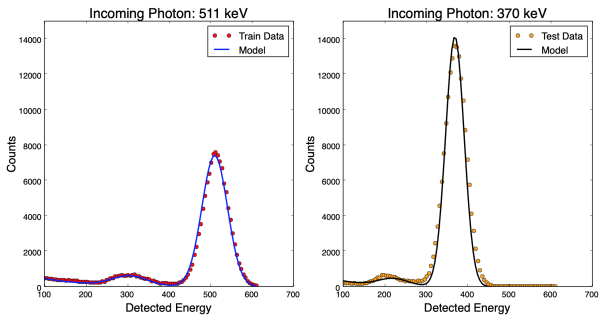

Fig. 2. Proposed detection efficiency model: training-set (511 keV point source emission positioned at the centre of the scanner) and testing-set (370 $\mathrm{keV}$ point source emission positioned at the centre of the scanner).

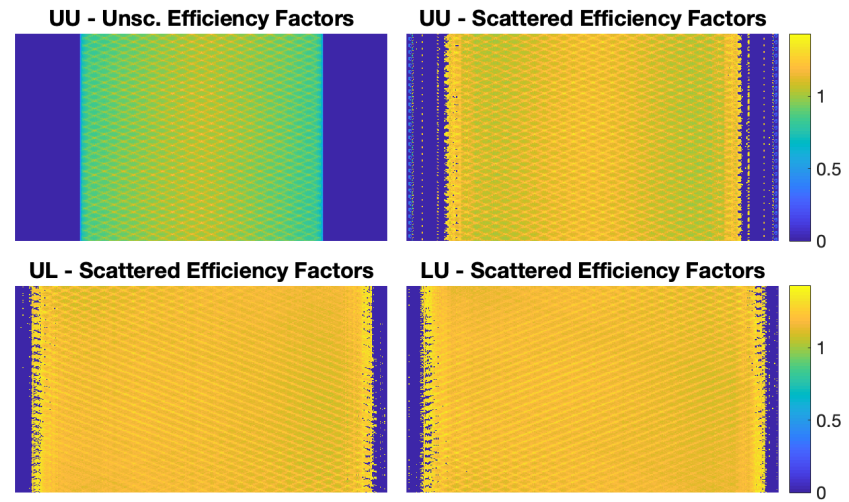

Fig. 3. Efficiency factors sinograms obtained from the cylindrical phantom for the energy windows: UU, UL and LU. Sinograms at all axial positions were summed for display purposes.

\section{B. Estimation of Normalisation Factors}

Results in Fig. 3 demonstrate that the geometric effects vary significantly between the photopeak and lower windows. As scatter events show a relatively high variation of the probability of angles of incidence and points of origination; the resulting normalisation sinograms look more homogeneous and flat in the low energy windows, with a less marked 'end block efficiency-drop' (diagonal stripes on the sinogram). Furthermore, the UL and LU normalisation sinograms show a mirror symmetry; this corresponds to the fact that only one of the photons is scattered, and therefore the geometric effect appears more 'blurred' due to the scattered photon only.

\section{XCAT Simulation - Comparison in Sinogram Space}

Sinograms from all energy window pairs are compared in Fig. 4, showing that the proposed forward model matches closely Monte Carlo simulations. The photopeak data show the best match amongst the three sinograms, whilst the low energy windows slightly overestimate the number of scatter events; a heuristic scaling factor was found to be needed to match more closely the GATE simulation (Sec. IV-F1).

\section{XCAT Reconstruction of activity and attenuation}

Fig. 5 shows the reconstruction error in the activity and attenuation images from GATE Monte Carlo generated data. The reconstruction was computed with and without scaling factor (Sec. IV-F1). The reconstruction from UU data only are also shown; aMLAA-S (Sec. III-B2a), LBFGS-AC (Sec. III-B2b) and LBFGS-MRAC (Sec. III-B2c) outputs are reported.

Results show that errors in the forward model propagate in the estimated activity and attenuation maps. In particular, effects of remaining model mismatch on the final estimates seem to arise in the region outside the lung - consistent in all the three reconstructions. With regards to the lung region, the best solution (closer to the one from LBFGS-AC) is achieved with aMLAA-EB-S (when a scaling factor is incorporated in the normalisation) and aMLAA-S.

Mean values in ROI (Fig. 6) confirm that reconstruction errors from aMLAA-EB-S (with $\zeta=0.93$ ) and aMLAA-S appear similar, whilst aMLAA-EB-S (with $\zeta=1$ ) shows a higher error in both attenuation and activity estimation.

\section{DISCUSSION}

This study investigated the problem of low-energy window normalisation and the effect of input/model mismatch on the multiple energy window joint reconstruction.

The benefits of incorporating low-energy photons information in PET image reconstruction have been discussed in [19], [31], [32]. Previous studies [15], [19] based on relatively simplistic analytical simulations have also shown that the multiple energy window reconstruction outperforms the single energy window approach. However, the behaviour of this methodology for the case of realistic measurements was yet unknown.

To accurately predict the expected measurements, an appropriate model for the detector energy response is needed [33]. In particular, results from this manuscript show that both the energy-dependent efficiency model and the normalisation technique play an important role in the accuracy of the forward model for quantitative measurements. In particular, the detection sensitivity of the low energy window was found to be significantly different from that of the photopeak window.

The output of aMLAA-EB-S from GATE Monte Carlo simulated data was compared to the joint reconstruction output from a single energy window, aMLAA-S. Fig. 5 shows that aMLAA-EB-S with $\zeta=0.93$ achieved a final error in the activity estimate comparable to the one from an LBFGSAC reconstruction. On the other hand, the aMLAA-EB-S reconstruction with $\zeta=1$ shows a final negative bias. Results from aMLAA-S were also in good agreement with LBFGSAC.

Together with the fact that aMLAA-EB-S was shown to be sensitive to errors in the forward model, this raises the question of whether the use of multiple energy windows will be beneficial in practice. According to the results presented in this manuscript, some aspects would favour a practical application of aMLAA-S compared to aMLAA-EB-S: (i) lower computational burden, (ii) no real need to accurately model detector scatter - for a standard single energy window acquisition -, (iii) no need to acquire data in multiple energy windows. However, this has not been tested for small structures and it could represent the objective of future investigations. Moreover, aMLAA-EB-S brings additional benefits due to the lower variance in the estimated images (shown in 

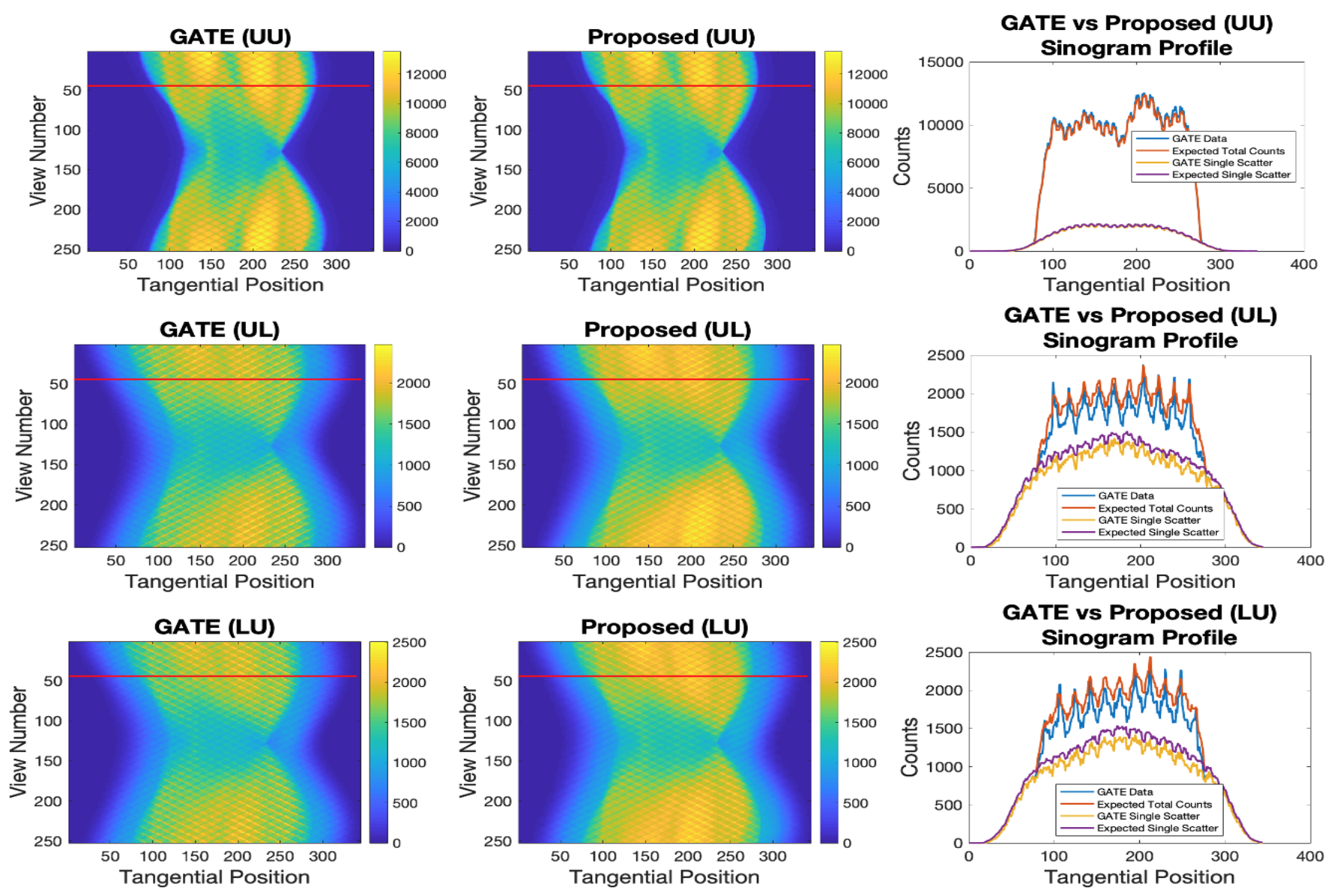

Fig. 4. Comparison in sinogram space between Monte Carlo GATE simulation and analytical 3D XCAT torso simulation. First row: UU data. Second row: UL data. Third row: LU data. First column: GATE Monte Carlo generated data. Second column: proposed analytical generated data. Sinograms at different axial positions were summed for display purposes. Third column: Sinogram profile comparison between GATE and the proposed method.

[15]) for a sufficiently accurate forward model. Furthermore, using multiple energy windows could allow the possibility of accounting for higher scattering angles in the model, giving the chance to directly model out-of-FOV scatter. Given the lower computational cost, one practical option could be to use aMLAA-S for the initial iterations and then use aMLAA-EB$\mathrm{S}$ to further refine the solution. This hypothesis will require further investigation.

One other aspect to mention is that aMLAA-EB-S and aMLAA-S rely on an alternating approach, meaning that optimal settings of inner loops need to be established. An alternating approach was chosen given the different intensity scales of the activity and attenuation gradients and different levels of numerical conditioning of the different contributing loglikelihoods. One possible way to avoid the alternating scheme could be to use the simultaneous approach (as in [15]) and to include pre-conditioning, similar to [30], [34]. Furthermore, the frequency at which the photopeak scatter is updated could be reduced over iterations to decrease computational cost. This will however require additional testing and validation.

For the single energy window reconstruction, the penalty strength in both attenuation and activity updates was reduced according to the global scale of the likelihood $\left(\mathcal{L}_{U U}\right.$ vs $\left.\mathcal{L}_{\mathrm{UU}}+\mathcal{L}_{\mathrm{UL}}+\mathcal{L}_{\mathrm{LU}}\right)$ computed from the ground truth dis- tributions. However, comparing reconstructions from different cost functions and penalty strengths is challenging. Further assessments are required in this regard, such as varying the penalty strengths and investigating multiple noise realisations. It is a possibility that the accuracy of the reconstruction could also vary depending on the chosen penalty term. Furthermore, the accuracy of the PLS depends on the reliability of the MR information. Different penalty terms could also be investigated. In addition to this, a fine tuning of the penalty would be needed to reduce the noise in the final attenuation estimates from both aMLAA-EB-S and aMLAA-S.

As the scatter distribution varies with the size and shape of an object, concerns arise on the dependency of the scatter efficiency factors on the geometry of the phantom used for computing the normalisation. In this study, this effect was partially overcome by using a global factor $\zeta$ adjusting the relative scale between the photopeak and UL/LU windows (Fig. 4). This would not be applicable for reconstructing real data acquisitions; improving on the efficiency model (Appendix A) and using an object matching more closely an actual patient density distribution to determine the normalisation factors should give better results. Possibly, a cylindrical phantom with 'lung' inserts could improve on the final normalisation sinogram used for thorax imaging. This hypothesis, however, 


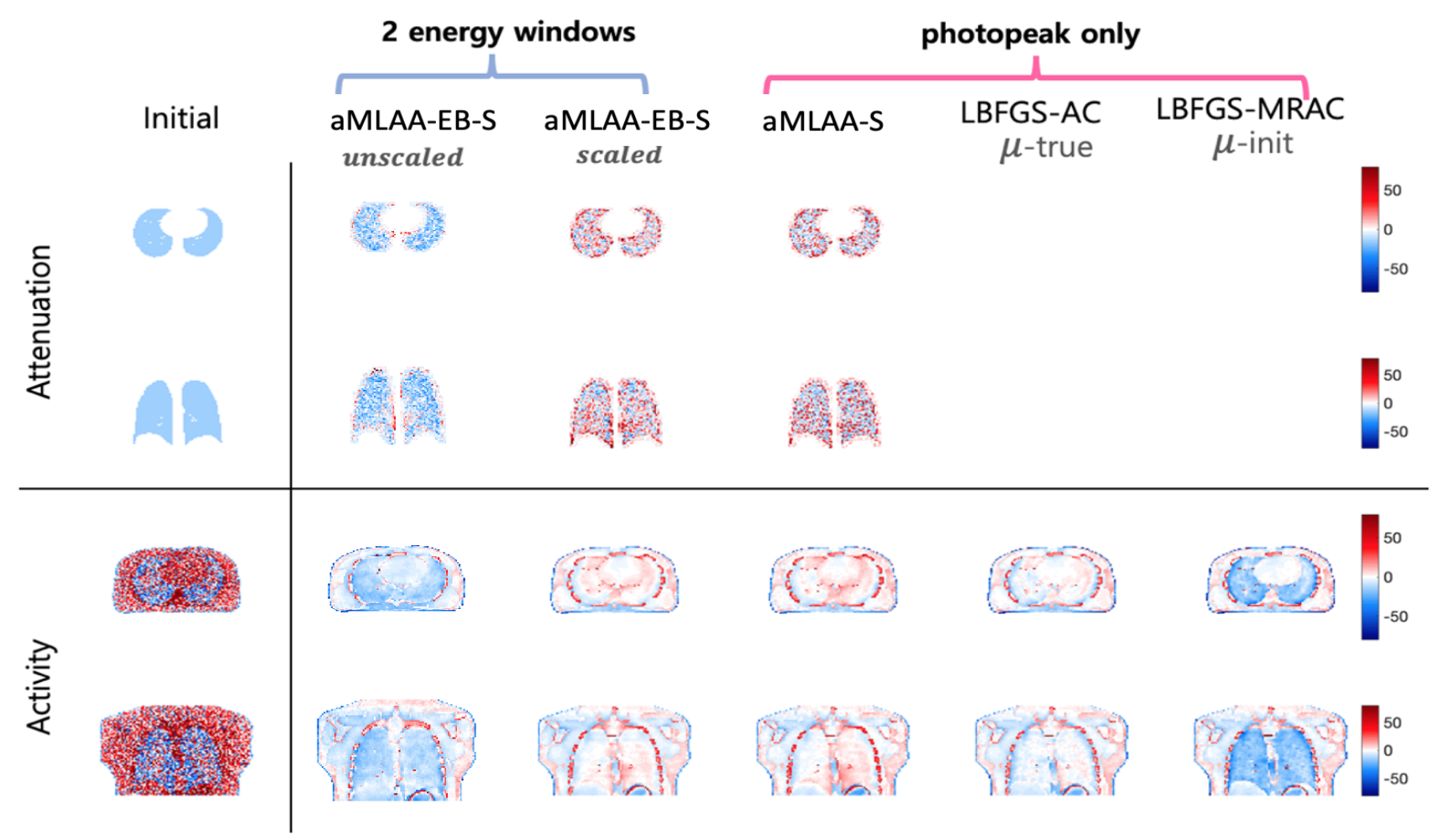

Fig. 5. Reconstruction error from GATE Monte Carlo generated data. First and second row: error in the attenuation. Third and fourth row: error in the activity image. First column: initial error (reconstruction input). Second column: reconstruction error from aMLAA-EB-S with $\zeta=1$. Penalty strengths: $\beta_{\boldsymbol{\mu}}=80$ and $\beta_{\boldsymbol{\lambda}}=5$. Third column: reconstruction using from aMLAA-EB-S with $\zeta=0.93$. Fourth column: reconstruction from aMLAA-S. Penalty strengths: $\beta_{\boldsymbol{\mu}}=60$ and $\beta_{\boldsymbol{\lambda}}=3$. Fifth column: emission reconstruction from LBFGS-AC. Penalty strength: $\beta_{\boldsymbol{\lambda}}=3$. Sixth column: emission reconstruction from LBFGS-MRAC (using the initial attenuation image). Penalty strength: $\beta_{\boldsymbol{\lambda}}=3$.
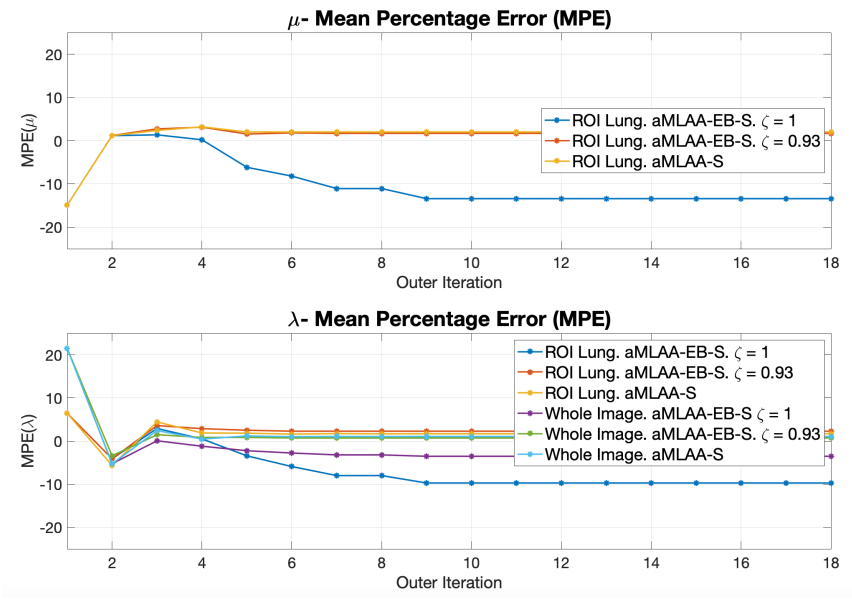

Fig. 6. Mean percentage Error (MPE) of the ROI values over iterations in the reconstructed attenuation (top) and activity (bottom) image.

will need to be confirmed with further investigations.

Assessing the feasibility of this methodology on patient data would bring additional value to this proposed method. However, to make this approach applicable in clinics, further efforts would be required. First, there is a need to validate the proposed detection efficiency model on real measurements from clinical scanners. Second, the necessity to account for multiple scatters and out-of-FOV scatters, and to further improve on the normalisation method. In particular, the latter relies on the possibility of differentiating between scattered and unscattered events. One possible solution for clinical application could be the following. First, the unscattered count efficiency factors could be obtained from a thin moving line source as in [35]. Once obtained, the true count sensitivity could be incorporated in the forward model so that the only unknown sensitivity is the one related to the scatter events. The scatter sensitivity $\boldsymbol{\Psi}_{v w}^{\mathrm{sc}}$ could be estimated from the total measured events. This method will need to be tested in the future.

Our results have shown how different energy windows require different normalisation sinograms given the wide range of angle of incidence of the incoming photons; the presence of gaps between detector blocks will likely make this effect larger, motivating even more the need of an energy-dependent detection efficiency model.

It is important to mention that with out-of-field activity and presence of double-scatter, the current heuristic scaling correction is likely not to be adequate. The model mismatch analysis is limited in this respect.

Multiple scatter estimation [16], [36] could be however incorporated into our algorithm using the same strategy as for the single scatter estimation in the photopeak window (onestep-late approach). However, this will need existing multiple scatter estimation code to be adapted to use multiple energy windows, and the accuracy of the existing multiple scatter models for low-energy windows to be established. 
Out-of-FOV scatters could be also accounted for by our method as the only constraint on the scatter locations is given by the boundaries of the attenuation map. The template image given to the algorithm could be extended beyond the FOV to place scatter locations outside the edge of the scanner. Similarly to how the arm regions were recovered in [37], the methods proposed in this manuscript that take scatter into account for the joint reconstruction might allow estimating activity and attenuation elsewhere, albeit most likely at low spatial resolution.

In this manuscript, the attenuation update was restricted to the lung region. Among the different tissue classes defined in standard MRAC methods, the lungs have the largest variability of inter-patient attenuation values [38]. Therefore, this assumption is fairly common in PET/MR studies [4], [38]. However, this assumption could be relaxed, potentially with the help of the incorporation of prior information.

This study is restricted to direct planes only. The 3D nature could potentially benefit the joint problem as it introduces correlations. This will apply to both aMLAA-EB-S and aMLAA$S$ but possibly favour aMLAA-EB-S over aMLAA-S due to scatter between-planes. However, efforts should be made in order to reduce the computational burden led by higher dimension sinograms.

Extending our method to TOF-PET would represent an interesting direction of future research. Without taking scatter into account, TOF information improves on the poor conditioning of the problem, but comes with the main limitation of a global scaling factor in the estimated activity distribution [8]. From previous studies in [13] and our current results, it appears that the scatter information could solve the scaling issues of TOF-MLAA, but this needs to be confirmed in future research studies.

Finally, the possibility of improving on the reconstruction methods discussed in this publication by using more than two energy windows should be investigated. Both the theoretical framework of the proposed methods and their implementations are suitable for this extension. However, the computational complexity of the algorithm scales quadratically with the number of energy windows. Furthermore, an increased number of normalisation sinograms will be needed to be computed. Therefore, an optimal value for the number of energy windows should be found where the computational cost in the algorithm is offset by the quantitative accuracy of the result.

\section{CONCLUSION}

This work investigated the feasibility of joint reconstruction of the activity and attenuation distributions of 3D objects from multiple energy window measurements by using a maximum likelihood framework in a realistic setting.

As low energy windows see a higher number of low energy photons, for which geometric factors differ markedly, the necessity for new model-based corrections for the normalisation of PET emission data from multiple-energy windows was investigated in this manuscript.

This work eliminates the dependency of over-simple assumptions of other previous work, leading to the possibility of reconstructing 3D volumes with data simulated for a realistic clinical PET/MR system in the presence of input/model mismatch and considering the presence of detector scatter.

Results show that with the proposed model, our analytic simulations correspond in good approximation to the Monte Carlo simulations, with only small deviations. The effect of such error on the final reconstructed attenuation and activity distributions was investigated. The multiple energy window approach was found to be sensitive to input/model mismatch but capable of leading to accurate attenuation and activity estimates for a sufficiently accurate forward model, in agreement with previous results based on analytical simulations only.

This manuscript has progressed this technique from being a simplistic idea to a reconstruction method able to reconstruct Monte Carlo generated data. This constitutes a significant step towards real data application in PET imaging of this type of methodology. Investigating the possibility of translating this approach to clinical practice remains the objective of future work.

\section{ACKNOWLEDGEMENT}

The authors would like to thank Dr. Charles C. Watson, Dr. Paul Schleyer (Siemens Healthineers) and Dr. Bjoern Jakoby (Siemens Healthineers) for helpful discussions.

\section{APPENDIX A}

\section{Detection EFFICIENCY Model}

Detector response varies as a function of the energy of the incoming photon. A semi-empirical response function for photon energies between 0.5 and $1.5 \mathrm{MeV}$ was recently proposed [39]. This model was adapted in this manuscript to the case of LSO detectors (the detector type used in the Siemens mMR) and to PET energy ranges. The proposed model is described in the following.

Similarly to the case of the photopeak window, the detection probability is obtained by integrating the probability of detecting the energy response function $f\left(E, E_{m}\right)$ between the boundaries of the energy window $t_{L}$ and $t_{H}$ :

$$
\epsilon\left(E, t_{L}, t_{H}\right)=\int_{t_{L}}^{t_{H}} f\left(E, E_{\mathrm{m}}\right) \mathrm{d} E_{m}
$$

Please note that $E$ and $E_{m}$ indicate respectively the incoming and measured photon energy. The detection efficiency model $f\left(E, E_{\mathrm{m}}\right)$ is given by the sum of four contributions, of which the following paragraphs give an overview:

$$
\begin{gathered}
f\left(E, E_{m}\right)= \\
f_{1}\left(E, E_{m}\right)+f_{2}\left(E, E_{m}\right)+f_{3}\left(E, E_{m}\right)+f_{4}\left(E, E_{m}\right)
\end{gathered}
$$

Pair production was not included in the model given the almost zero probability of this effect occurring for incoming photon energies of $511 \mathrm{keV}$ (or lower).

\section{A. Photoelectric effect:}

A Gaussian distribution function is adopted to describe the detection efficiency for a gamma photon whose energy is completely deposited: 


$$
f_{1}\left(E, E_{m}\right)=\frac{\sigma_{\mathrm{T}}}{\sqrt{2 \pi \sigma_{\mathrm{P}}}} \exp \left(\frac{-\left(E_{m}-E\right)^{2}}{2 \sigma_{\mathrm{P}}^{2}}\right)
$$

where $\sigma_{\mathrm{P}}$ is the standard deviation of the photopeak. The amplitude of the peak is proportional to the total photoelectric cross section $\sigma_{\mathrm{T}}$, given by:

$$
\sigma_{\mathrm{T}}=\frac{Z_{\mathrm{eff}}{ }^{5}}{E}
$$

For lower energy photons, the photoelectric cross section increases, and the amplitude of the photopeak is therefore expected to be higher. The expression $Z_{\text {eff }}$ indicates the atomic number of hypothetical single element that would attenuate photons at the same rate as the composite material in question [40]. The (effective) atomic number $Z_{\text {eff }}$ was set to 66 , corresponding to that of LSO crystal.

\section{B. Compton Scattering:}

To model detector scatter the function proposed by [39] was modified here by incorporating the dependency on the total Compton cross section $\sigma_{\mathrm{C}}$ and the effective atomic number $Z_{\text {eff }}$. The resulting function becomes:

$$
\begin{gathered}
f_{2}\left(E, E_{m}\right)= \\
H_{2} Z_{\text {eff }} \sigma_{\mathrm{C}}\left[\left(\frac{E}{E^{\prime}}\right)+\left(\frac{E^{\prime}}{E}-2\right)\right] \exp \left(\frac{-\left(E_{m}-p_{1} E\right)^{2}}{4 p_{2} \sigma_{\mathrm{P}}}\right)
\end{gathered}
$$

where $E^{\prime}$ is the energy after Compton scatter with $\phi=\pi, H_{2}$, $p_{1}$ and $p_{2}$ are parameters to scale the amplitude, determine the centre of the Compton plateau and its standard deviation, respectively.

\section{Flat continuum:}

An 'almost' flat continuum from zero to the photopeak energy found to exist in the spectrum [39], possibly resulting from the presence of electronic noise. It is modelled as:

$$
f_{3}\left(E, E_{m}\right)=H_{3} \operatorname{erfc}\left(\frac{E_{m}-E}{\sqrt{2} \sigma_{\mathrm{P}}}\right)
$$

where $H_{3}$, is a parameter to scale the amplitude of the flat continuum. For $E>E_{m}, f_{3}\left(E, E_{m}\right)=0$ and erfc $=1-$ erf.

\section{Exponential tail:}

This last function relates to the presence of an exponential tail in the region of low energy side of the spectrum. The representative function is given by:

$$
\begin{aligned}
& f_{4}\left(E, E_{m}\right)= \\
& H_{4} \exp \left(\frac{E_{m}-E}{\sqrt{2} \pi p_{3} \sigma_{\mathrm{P}}}\right) \operatorname{erfc}\left(\frac{E_{m}-E}{\sqrt{2} \sigma_{\mathrm{P}}}+\frac{1}{2 p_{3}}\right)
\end{aligned}
$$

where $H_{4}$ and $p_{3}$ are parameters that scale the amplitude, the standard deviation and the slope of the exponential tail, respectively.

\section{APPENDIX B \\ Single SCATTER Model}

The scatter forward model used in this manuscript is formulated as:

$$
\begin{gathered}
\tilde{g}_{v w i j s}^{\mathrm{sc}}(\tilde{\boldsymbol{\theta}})= \\
\frac{1}{\alpha}\left(\boldsymbol{\Psi}_{v w}(E, 511) \mathrm{I}_{i s j}(\tilde{\boldsymbol{\theta}})+\mathbf{\Psi}_{v w}(511, E) \mathrm{I}_{j s i}(\tilde{\boldsymbol{\theta}})\right)
\end{gathered}
$$

where $E$ is the photon energy after (single) Compton scattering (in $\mathrm{keV}$ ) as a function of the scatter angle $\varphi$ for a scatter point at voxel $s, \alpha=\frac{3}{4} \frac{\sigma_{i} \sigma_{j}}{2 \pi r^{2}}$ approximates the solid angle at each detector $i j, \boldsymbol{\Psi}_{v w}(511, E)$ being the matrix of efficiency factors for a scatter coincidence and:

$$
\begin{gathered}
\mathrm{I}_{i s j}(\tilde{\boldsymbol{\theta}})= \\
\frac{\sigma_{i s} \sigma_{j s}}{4 \pi r_{i s}^{2} r_{j s}^{2}} \frac{d \sigma}{d \Omega}(\varphi) \mu_{511, s}\left(\boldsymbol{K}_{i s} \tilde{\boldsymbol{\lambda}}\right) \mathrm{e}^{-\boldsymbol{K}_{i s} \tilde{\boldsymbol{\mu}}_{511} \mathrm{e}^{-\boldsymbol{K}_{j s} \tilde{\boldsymbol{\mu}}_{E}}}
\end{gathered}
$$

where $\frac{d \sigma}{d \Omega}(\varphi)$ is the differential cross section given by the Klein-Nishina $(\mathrm{KN})$ equation [41], $\tilde{\boldsymbol{\mu}}_{E}$ is the distribution of attenuation coefficients at photon energy $\mathrm{E}, r_{i s}$ is the distance between the scatter point $s$ and detector $i, \sigma_{i s}$ denotes the detector cross-section presented to the ray $i s$, and $\boldsymbol{K}_{i s}$ indicates the line integral operator along the line $i s$ [20].

1) Probability of scattering: The $\mathrm{KN}$ formula in (25) predicts the number of photons that will scatter with a certain angle $\varphi$, which can be also related to the change in photon energy via the Compton equation. The final energy of the scattered photon only depends on the scattering angle and on the original photon energy.

2) Effect of reduced energy on attenuation after Compton scattering: The Compton scattering process results in the reduction of the photon energy as well as change in direction. As a result, photon attenuation after the point of scatter is increased. Here we address the dependency of the attenuation on the energy in the scatter model by assuming that the attenuation is only due to Compton scatter and is therefore proportional to the total Compton scatter cross-section $\sigma_{\text {tot }}$ at a particular energy $E$ [15]:

$$
\boldsymbol{\mu}_{E}=\frac{\sigma_{\mathrm{tot}}(\Lambda)}{\sigma_{\mathrm{tot}}(1)} \boldsymbol{\mu}_{511}
$$

where $\sigma_{\text {tot }}$ can be obtained by integrating $\mathrm{KN}$ over all solid angles and $\Lambda=\frac{E}{511}$. The forward model can therefore be rewritten with respect to the attenuation coefficient at reference energy $(511 \mathrm{keV})$.

\section{APPENDIX C \\ GRADIENT OF THE OBJECTIVE FUnCTION}

To be able to solve (8) it is required to compute its gradient $\nabla \mathcal{L}_{\boldsymbol{\theta}}^{\text {tot }}=\sum_{(v, w)} \nabla \mathcal{L}_{\mathrm{v}, \mathrm{w}}$, where:

$$
\nabla_{\boldsymbol{\theta}} \mathcal{L}_{v w}=\left(\boldsymbol{J}_{v w}^{\mathrm{unsc}}+\boldsymbol{J}_{v w}^{\mathrm{sc}}\right)^{\top}\left(\boldsymbol{g}_{v w} \oslash \overline{\boldsymbol{g}}_{v w}-\mathbf{1}\right)
$$

with $\oslash$ indicating the element-wise division, (.. $)^{\top}$ indicating the transpose operator, and 1 being a vector of ones.

Dropping the energy window indices $v w$, the Jacobian $\boldsymbol{J}^{\text {unsc }}=\left[\boldsymbol{J}_{\lambda}^{\text {unsc }}, \boldsymbol{J}_{\mu}^{\text {unsc }}\right]$ is given by : 


$$
\begin{gathered}
\boldsymbol{J}_{\lambda}^{\text {unsc }}=\boldsymbol{\Psi} \boldsymbol{A}(\boldsymbol{\mu}) \\
\boldsymbol{J}_{\mu}^{\text {unsc }}=-\boldsymbol{\Psi} \boldsymbol{D}(\boldsymbol{A}(\boldsymbol{\mu}) \boldsymbol{\lambda}) \boldsymbol{L}
\end{gathered}
$$

The Jacobian $\boldsymbol{J}^{\mathrm{sc}}=\left[\boldsymbol{J}_{\lambda}^{\mathrm{sc}}, \boldsymbol{J}_{\mu}^{\mathrm{sc}}\right]$ is given by :

$$
\begin{aligned}
& \boldsymbol{J}_{\boldsymbol{\mu}}^{\mathrm{sc}}=\boldsymbol{P} J_{\tilde{\mu}}^{\mathrm{sc}} \boldsymbol{R} \\
& \boldsymbol{J}_{\boldsymbol{\lambda}}^{\mathrm{sc}}=\boldsymbol{P} \boldsymbol{J}_{\tilde{\boldsymbol{\lambda}}}^{\mathrm{sc}} \boldsymbol{R}
\end{aligned}
$$

with $\boldsymbol{P}$ and $\boldsymbol{R}$ defined in II-A. Please note that an expression for $\boldsymbol{J}_{\tilde{\boldsymbol{\lambda}}}^{\mathrm{sc}}$ and $\boldsymbol{J}_{\tilde{\boldsymbol{\mu}}}^{\mathrm{sc}}$ was given in [15].

\section{REFERENCES}

[1] H. Jadavar and P. M. Colletti, "Competitive Advantage of PET/MR," Eur J Radiol, vol. 83, no. 1, pp. 84-94, 2013.

[2] J. H. Jung and K. C. Im, "PET/MRI: Technical Challenges and Recent Advances," Nuclear Medicine in Molecular Imaging, vol. 50, no. 1, pp. $3-12,2016$.

[3] A. Mehranian, H. Zaidi, and A. J. Reader, "MR-guided joint reconstruction of activity and attenuation in brain PET-MR," NeuroImage, vol. 162, pp. 276-288, 2017.

[4] Y. Berker, A. Salomon, F. Kiessling, and S. Volkmar, "Lung Attenuation Coefficient Estimation Using Maximum Likelihood Reconstruction of Attenuation and Activity for PET/MR Attenuation Correction," IEEE NSS/MIC Conference Record, 2012.

[5] J. Lillington, L. Brusaferri, K. Kläser, K. Shmueli, R. Neji, B. F. Hutton, F. Fraioli, S. Arridge, M. J. Cardoso, S. Ourselin, K. Thielemans, and D. Atkinson, "PET/MRI attenuation estimation in the lung: A review of past, present, and potential techniques," Medical Physics, vol. 47, no. 2, pp. $790-811,2020$.

[6] V. Cuplov, B. F. Holman, J. McClelland, M. Modat, B. F. Hutton, , and $\mathrm{K}$. Thielemans, "Issues in quantification of registered respiratory gated PET/CT in the lung," Physics in Medicine and Biology, vol. 63, no. 1, 2017.

[7] J. Nuyts, P. Dupont, S. Stroobants, S. Benninck, L. Mortelmans, and P. Suetens, "Simultaneous maximum a posteriori reconstruction of attenuation and activity distributions from emission sinograms," IEEE Transaction on Medical Imaging, vol. 18, no. 5, pp. 393-403, 1999.

[8] M. Defrise, A. Rezaei, and J. Nuyts, "Time-of-flight PET data determine the attenuation sinogram up to a constant," Physics in Medicine and Biology, vol. 57, pp. 885-899, 2012.

[9] A. Rezaei, S. Schramm, S. M. A. Willekens, S. Delso, K. V. Laere, and J. Nuyts, "A Quantitative Evaluation of Joint Activity and Attenuation Reconstruction in TOF-PET/MR Brain Imaging," Journal of Nuclear Medicine, 2019.

[10] Y. Berker and Y. Li, "Attenuation correction in emission tomography using the emission data - A review," Medical Physics, vol. 43, no. 2, pp. 807-832, 2016.

[11] S. C. Cade, S. Arridge, M. J. Evans, and B. F. Hutton, "Use of measured scatter data for the attenuation correction of single photon emission tomography without transmission scanning," Medical Physics, vol. 40, no. 8, p. 082506 (12pp.), 2013.

[12] A. Bousse, A. Sidlesky, N. Roth, A. Rashidnasab, and K. Thielemans, "Joint Activity/Attenuation Reconstruction in SPECT Using Photopeak and Scatter Sinograms," IEEE NSS/MIC Conference Record, 2016.

[13] Y. Berker, F. S. Kiessling, and V. Schulz, "Scattered PET data for attenuation-map reconstruction in PET/MRI," Medical Physics, vol. 41, no. 10, p. 102502, 2014.

[14] Y. Berker, J. S. Karp, and V. Schulz, "Numerical Algorithms for Scatter-to-Attenuation Reconstruction in PET: Empirical Comparison of Convergence, Acceleration, and the Effect of Subsets," IEEE Transaction on Radiation and Plasma and Medical Sciences, vol. 1, no. 15, pp. 426434, 2017.

[15] L. Brusaferri, A. Bousse, E. C. Emond, R. Brown, Y.-J. Tsai, D. Atkinson, C. C. Watson, B. F. Hutton, S. Arridge, and K. Thielemans, "Joint Activity and Attenuation Reconstruction from Multiple Energy Window Data with Photopeak," IEEE Transactions on Plasma and Radiation Medical Sciences, vol. 4, no. 4, pp. 410-421, 2020.

[16] C. Tsoumpas, P. Aguiar, D. Ros, N. Dikaios, and K. Thielemans, "Scatter Simulation Including Double Scatter," IEEE NSS/MIC Conference Record, 2005.

[17] C. C. Watson, "Extension of Single Scatter Simulation to Scatter Correction of Time-of-Flight PET," IEEE Transaction on Nuclear Science, vol. 54, no. 5, pp. 1679-86, 2007.
[18] J. M. Ollinger, "Detector Efficiency and Compton Scatter in Fully 3D PET," IEEE Trans. On Nucl. Sci., vol. 42, no. 4, pp. 1168-1173, 1995.

[19] Y. Berker, V. Schulz, and J. S. Karp, "Algorithms for joint activity-attenuation estimation from positron emission tomography scatter," EJNMMI Physics, vol. 6, no. 18, 2019.

[20] C. C. Watson, D. Newport, and M. E. Casey, "A single scatter simulation technique for scatter correction in 3D PET," 3D image Reconstruction in Radiation and Nuclear Medicine, pp. 256-268, 1996.

[21] J. M. Ollinger, "Model-based scatter correction for fully 3D PET," Physics in Medicine and Biology, vol. 41, no. 1, pp. 153-76, 1996.

[22] D. Hogg, K. Thielemans, T. Spinks, and N. Spyrou, "Maximumlikelihood estimation of normalisation factors for PET," IEEE NSS/MIC Conference Record, vol. 4, 2001.

[23] R. H. Byrd, P. Lu, J. Nocedal, and C. Zhu, "A Limited Memory Algorithm for Bound Constrained Optimization," SIAM Journal of Scientific Computing, vol. 16, no. 5, pp. 1190-1208, 1995.

[24] M. J. Ehrhardt, P. Markiewicz, M. Liljeroth, A. Barnes, V. Kolehmainen, J. S. Duncan, P. L., D. Atkinson, B. F. Hutton, S. Ourselin, K. Thielemans, and S. R. Arridge, "PET Reconstruction With an Anatomical MRI Prior Using Parallel Level Sets," IEEE Transaction on Medical Imaging, vol. 35, no. 9, pp. 2189-2200, 2016.

[25] A. M. Karlberg, O. Sæther, L. Eikenes, and P. E. Goa, "Quantitative comparison of PET performance-Siemens biograph mCT and mMR," EJNMMI Physics, vol. 3, no. 5, p. 1, 2016.

[26] S. Jan, G. Santin, D. Strul, S. Staelens, K. Assie, D. Autret, S. Avner et al., "GATE: A simulation toolkit for PET and SPECT," Physics in Medicine and Biology, vol. 49, no. 49, pp. 14543-4561, 2004.

[27] K. Thielemans, C. Tsoumpas, S. Mustafovic, T. Beisel, P. Aguiar, N. Dikaios, and M. W. Jacobson, "STIR: Software for tomographic image reconstruction release 2," Physics in Medicine and Biology, vol. 57, no. 4, pp. 867-883, 2012.

[28] P. Virtanen, R. Gommers, T. E. Oliphant et al., "SciPy 1.0-Fundamental Algorithms for Scientific Computing in Python," arXiv e-prints, $\mathrm{p}$. arXiv:1907.10121, 2019.

[29] W. P. Segars, G. Sturgeon, S. Mendonca, J. Grimes, and B. M. Tsui, "4D XCAT phantom for multimodality imaging research," Medical Physics, vol. 37, no. 9, pp. 4902-15, 2010.

[30] Y.-J. Tsai, P. Lu, A. Bousse, M. J. Ehrhardt, C. W. Stearns, S. Ahn, B. Hutton, S. Arridge, and K. Thielemans, "Fast Quasi-Newton Algorithms for Penalized Reconstruction in Emission Tomography and Further Improvements via Preconditioning," IEEE Transaction on Medical Imaging, vol. 37, no. 4, pp. 1000-1010, 2017.

[31] M. Conti, I. Hong, and C. Michel, "Reconstruction of scattered and unscattered PET coincidences using TOF and energy information," Physics in Medicine and Biology, vol. 57, pp. N307-N317, 2012.

[32] L. Brusaferri, A. Bousse, Y.-J. Tsai, D. Atkinson, S. Ourselin, B. F. Hutton, S. Arridge, and K. Thielemans, "Maximum-likelihood estimation of emission and attenuation images in 3D PET from multiple energy window measurements," IEEE NSS/MIC Conference Record, 2018.

[33] L. Brusaferri, "Improving quantification in non-TOF 3D PET/MR by incorporating photon energy information," PhD Thesis, 2020.

[34] N. Fuin, S. Pedemonte, A. O. Catalano, D. Izquierdo-Garcia, A. Soricelli, M. Salvatore, K. Heberlein, J. M. Hooker, K. V. Leemput, and C. Catana, "PET/MRI in the presence of metal implants: Completion of the attenuation map from PET emission data," Journal of Nuclear Medicine, vol. 58, pp. 840-845, 2017.

[35] R. D. Badawi, P. Marsden, B. F. Cronin, J. L. Sutcliffe, and M. N. Maisey, "Optimization of noise-equivalent count rates in 3D PET," Physics in Medicine and Biology, vol. 41, no. 9, pp. 1755-76, 1996.

[36] C. C. Watson, J. Hu, and C. Zhou, "Extension of the SSS PET scatter correction algorithm to include double scatter," IEEE NSS/MIC Conference Record, 2018.

[37] L. Brusaferri, A. Bousse, N. Efthimiou, E. Emond, D. Atkinson, S. Ourselin, B. F. Hutton, S. Arridge, and K. Thielemans, "Potential benefits of incorporating energy information when estimating attenuation from PET data," IEEE NSS/MIC Conference Record, 2017.

[38] A. Mehranian and H. Zaidi, "Emission-based estimation of lung attenuation coefficients for attenuation correction in time-of-flight PET/MR," Physics in Medicine and Biology, vol. 60, pp. 4813-4833, 2015.

[39] Z. Li et al., "A semi-empirical response function for Gamma-ray of Scintillation detector based on physical interaction mechanism," 2014.

[40] M. J. T. Birgani et al., "Determination of the effective atomic and mass numbers for mixture and compound materials in high energy photon interactions," Journal of Radioanalytical and Nuclear Chemistry, vol. 292, no. 3, p. 1367-1370, 2012. 
[41] O. Klein and Y. Nishina, "Uber die Streuung von Strahlung durch freie Elektronen nach der neuen relativistischen Quantendynamik von Dirac," Zeitschrift für Physik, vol. 52, no. 11-12, pp. 853-859, 1929. 